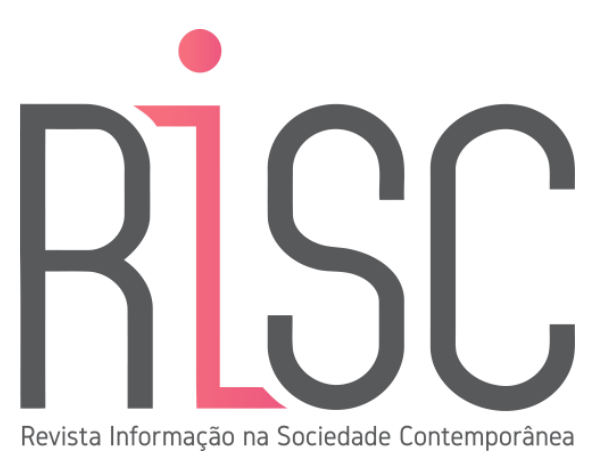

\title{
O FLUXO INFORMACIONAL DE FAKE NEWS BASEADAS EM DENÚNCIAS FALSAS PARA A DESTRUIÇÃO DA VIDA PRIVADA E PÚBLICA DE CIDADÃOS
}

\author{
Álvaro Maximiliano Pino Coviello* \\ Mestrando em Gestão da Informação \\ https://orcid.org/0000-0002-5528-144X E-mail: alvaropino@ufpr.br

\section{Rodrigo Eduardo Botelho-Francisco*} \\ Doutor em Ciência da Comunicação \\ (D) https://orcid.org/0000-0002-4772-9398 E-mail: rodrigobotelho@ufpr.br
}

* Universidade Federal do Paraná, Programa de Pós-Graduação em Gestão da Informação, Departamento de Ciência e Gestão da Informação, Curitiba, PR, Brasil.

\section{RESUMO}

Se procura caracterizar o fluxo informacional em dois casos de Fake News baseadas em denúncias falsas para a destruição da vida pública e privada de cidadãos. O fluxo informacional de Fake News é um objeto de estudo específico da Gestão da Informação. O fluxo é considerado importante porque quando as Fake News aparecem, a circulação com sua velocidade, densidade, variedade, localização e alcance pode levar as vítimas a tomar decisões desacertadas. Por outro lado, propõe abordar as consequências que as Notícias Falsas trouxeram para a vida dessas pessoas e conhecer o processo de tomada de decisão que elas tiveram em cada etapa do processo. Espera-se analisar as Fake News baseadas em 
denúncias falsas; descrever o fluxo informacional das mesmas; narrar a vida das vítimas nos diferentes estágios de circulação das Fake News; identificar a tomada de decisão das vítimas em todos os períodos. A pesquisa faz parte do Paradigma Construtivista, Naturalista e Interpretativo. Possui um olhar teórico interdisciplinar descritivo baseado na área da Informação e da Comunicação. Pretende-se conhecer certos ecossistemas comunicativos e entender uma situação problemática. A pesquisa espera contribuir para aprofundar a área de circulação de notícias falsas com um trabalho que retorne na sociedade para melhorar a vida dos cidadãos no sistema democrático.

Palavras-chave: Fluxo da Informação. Fake News. Denúncias Falsas. Vida pública e privada. Tomada de decisões.

\section{THE INFORMATIONAL FLOW OF FAKE NEWS BASED ON FALSE COMPLAINTS FOR THE DESTRUCTION OF PRIVATE AND PUBLIC LIFE OF CITIZENS}

\section{ABSTRACT}

It seeks to characterize the information flow in two cases of Fake News based on false accusations for the destruction of the public and private life of citizens. The informational flow of Fake News is an object of specific study of Information Management. The flow is considered important because when Fake News appears, circulation with its speed, density, variety, location and reach can lead victims to make wrong decisions. On the other hand, it proposes to address the consequences that False News brought to the lives of these people and to know the decision-making process they had at each stage of the process. It is expected to analyze Fake News based on false reports; describe their informational flow; narrate the lives of the victims at the different stages of Fake News circulation; identify victims' decision making at all times. The research is part of the Constructivist, Naturalistic and Interpretive Paradigm. It has a descriptive interdisciplinary theoretical look based on the area of Information and Communication. It is intended to know certain communicative ecosystems and understand a problematic situation. The research hopes to contribute to deepen the area of circulation of false news with work that returns to society to improve the lives of citizens in the democratic system.

Keywords: Information Flow. Fake News. False Complaints. Public and private life. Decision making.

\section{INTRODUÇÃO}

A escolha do tema parte especialmente de uma experiência pessoal do investigador, que teve que emigrar para o Brasil devido a uma campanha contra ele baseada em "Fake News", numa denúncia falsa que culminou com prejuízos irreparáveis em sua vida profissional e familiar. No campo profissional e acadêmico também é inegável o destaque e 
a importância que vem ganhando o tema na "Era da Informação", uma vez que as "Fake News" vêm sendo associadas a vários fatos da vida política cotidiana, vide as denúncias sobre o uso desta estratégia nas eleições americanas e brasileiras. No campo científico são diversas as áreas preocupadas com esta problemática. As Ciências da Comunicação, por exemplo, são desafiadas a estudar um novo ecossistema comunicativo, onde a desinformação e a má-informação ganha velocidade e se aproveita das características de notícia para circular, travestida de verídica, nos mídia. Na Ciência da Informação, por sua vez, preocupa não só a gênese desta representação da informação, bem como seu fluxo informacional e a necessidade de sua identificação e descarte.

A investigação visa conhecer o fluxo informacional de Fake News baseadas em estudo de caso da ocorrência de denúncias falsas na vida de dois sujeitos específicos; um, o próprio pesquisador, jornalista e professor; e outro, a chamada "Doutora da Morte" do Hospital Evangélico de Curitiba.

A pesquisa enfoca o fluxo informacional de Fake News, no âmbito específico da Gestão da Informação. Este olhar é considerado importante porque, quando as Fake News aparecem, a circulação com sua velocidade, densidade, variedade, localização e alcance influencia diretamente na tomada de decisões dos sujeitos impactados. Neste sentido, identificar cada etapa deste processo pode ser importante para as iniciativas que visam identificar e combater a circulação de notícias falsas, antes que elas ganhem alcance e impactem decisivamente a vida dos cidadãos. Para responder à questão, a pesquisa será delineada a partir de um objetivo geral, sendo: Descrever e caracterizar o fluxo informacional a partir de experiências pessoais com Fake News. Para tanto, tem como objetivos específicos: (i) Revisar os conceitos relacionados com as Fake News. (ii) Descrever o fluxo informacional das Fake News. (iii) Identificar o fluxo informacional das Fake News em experiências pessoais. (iv) Identificar pontos de tomada de decisão no fluxo informacional de Fake News.

\section{HIPÓTESE OU SUPOSIÇÕES DE PESQUISA}

A pesquisa faz parte do Paradigma Interpretativo, pela mesma razão, nenhuma hipótese é usada, mas suposições e perguntas de pesquisa. Isto é assim porque, de 
acordo com Sautu et al. (2005) a natureza da realidade a ser analisada é subjetiva e múltipla. O pesquisador está imerso no contexto que analisa e, embora não manipule variáveis, seus valores influenciam o trabalho. Uma suposição poderia ser: As características do fluxo informacional em dois casos pessoais impactados por Fake News poderiam ser semelhantes. Ou, dito de outra forma: O fluxo informacional em dois casos pessoais impactados por Fake News poderiam ter características semelhantes.

O concreto é que se precisa operar com os seguintes conceitos:

Da comunicação: - Ecossistemas comunicativos onde as pessoas impactadas estão inseridas. - Conteúdo das notícias falsas. - Aparição das notícias falsas: oportunidade temporal e espacial. - Canais de aparência. - Tempo de exposição das notícias. Viralização das notícias. - Temporalidade de aparência. - Gêneros, técnicas e tecnologias para desacreditar. - Fontes e autores ideológicos do descrédito do cidadão. -Reações dos consumidores das informações que foram registradas. - Ciclo de vida das Fake News: fluxo de quando aparece até ser descartada. - Radicação das denúncias falsas: fato concreto, atores, possíveis causas/ fundamentos do fato. - As ações da Justiça: nos fatos concretos e na estrutura legal sobre denúncias falsas e Fake News. -Biografia das vítimas: História da vida no momento do aparecimento das notícias falsas, história da vida após o aparecimento das notícias, história da vida após o pronunciamento da Justiça. - Nova análise do ecossistema comunicativo onde as vítimas estão inseridas.

Da informação: Os fatores que compõem o fluxo de informação de acordo a Inomata, Araújo e Varvakis (2015, p. 221) e Araújo, Silva e Varvakis (2017, p. 63) podem ser: - Atores: Todos os envolvidos no fluxo de informação, e nas atividades inerentes ao fluxo, e que, de alguma forma, são responsáveis para que o fluxo ocorra. - Canais: Responsáveis por suporte à transmissão de informações no processo de comunicação. Fontes de Informação: Insumo para a obtenção dos mais variados tipos de informação que darão suporte para as atividades que o fluxo de informação está inserido. - TICs: Suportes para que as operações do fluxo aconteçam de forma exequível. - Barreiras de acesso à informação: Entraves ocasionalmente encontrados no caminho que a informação deve percorrer. - Determinantes de escolha e uso: Aspectos que interferem na escolha da fonte e do uso da informação. - Necessidades informacionais: Fator responsável pelo início do processo e do fluxo de informação. - Velocidade de 
recuperação: Tempo de resposta entre as necessidades da informação e a resposta obtida.

O Paradigma Interpretativo permite trabalhar com perguntas de pesquisa:

No nível conceitual: O que é fluxo informacional? Que diálogo acadêmico por fluxo informacional pode ser estabelecido dentro do binômio informação-comunicação? Que são as Fake News - notícias falsas? Qual é o atual ecossistema comunicativo onde as Notícias Falsas aparecem? O que são as denúncias falsas? O que se entende por vida pública e privada? O que é um cidadão? O que se entende por direito à privacidade?

No nível operacional: Quais são as notícias falsas que se quer analisar? Quais são as denúncias falsas que deram origem ou apoiaram essas Fake News? Quais foram suas motivações? Como foi/é o ecossistema comunicativo onde as Notícias Falsas apareceram? Quais são as características do fluxo informacional das Notícias Falsas? Como o fluxo informacional pode ser visualizado no ecossistema comunicativo específico? Que repercussões as Notícias Falsas tiveram? Qual é a relação estabelecida entre fluxo informacional e repercussão? Quem são os sujeitos alvos da Fake News? Por que esses sujeitos são alvos a atingir? Como foi/ o comportamento e a vida dos sujeitos alvos? Que decisões esses sujeitos tomaram como resultado do fluxo informacional das Fake News? Embora o fluxo informacional das Notícias Falsas tenha produzido desinformação pública, para os sujeitos vítimas: o fluxo informacional thes forneceu informações para tomar decisões que avaliaram apropriadas? Ou sua tomada de decisão foi caracterizada pelo impulso dado pela própria mentira, de modo que a decisão foi equivocada? Qual é o momento atual do fluxo informacional das Fake News analisado no ecossistema comunicativo ao qual as vítimas pertencem? Existem características semelhantes do fluxo informacional entre esses dois casos?

No nível hipotético para pesquisas futuras: Que características comuns podem ter os fluxos informacionais para outros casos de Fake News baseadas em denúncias falsas? Essas características podem ser mensuradas? Se forem mensuráveis e repetitivas, o fluxo informacional e seu impacto pode ser predeterminado? Este nível não é desenvolvido, mas a pesquisa pode ser a base para trabalhos futuros ou no doutorado. 


\section{REFERENCIAL TEÓRICO}

Na Gestão da Informação, os fluxos de informação são os princípios vitais que suportam os processos, as tomadas de decisão, o desenvolvimento de produtos etc. Existem vários modelos dos autores clássicos que se devem aprofundar. Entre eles: 0 modelo de Choo (2003) atende o ciclo de conhecimento, no qual um fluxo contínuo de informações é mantido entre a criação de significado, a construção de conhecimento e a tomada de decisões, de maneira que o resultado do uso de informação em uma etapa ofereça um elaborado contexto, bem como mais recursos para o uso da informação nas outras etapas. O modelo de Davenport e Prusak (1998) representa a cooperação entre os atores envolvidos no fluxo de informação, que é a chave para que a informação possa circular na organização, chamados pelos autores de processo genérico de gerenciamento da informação. Esse processo é composto por quatro fases distintas: primeira fase: define quais informações são necessárias e para qual finalidade; segunda fase: define as estratégias de busca da informação e a implantação dessa obtenção; terceira fase: a informação obtida começa a circular na organização a fim de suprir as necessidades informacionais dos indivíduos; quarta fase: o uso da informação se apresenta como um processo cognitivo que abrange a recepção da informação pelo indivíduo, sua internalização e a sua transformação em conhecimento para resolver seus problemas informacionais. Valentim (2013a, 2013b) refere-se a ambientes organizacionais estáveis ou instáveis com tipos de fluxos horizontal, vertical e transversal. Também a partir Valentim (2020) se pode descrever o Ecossistema Comunicativo de Big Data que mudou as práticas profissionais do Jornalismo. Em Inomata, Araújo e Varvakis (2015, p. 221) e Araújo, Silva e Varvakis (2017, p. 63) por sua vez, se delimitaram os fatores que compõem o fluxo de informação de acordo com elementos (atores, canais, fontes e TICs) e aspectos de influência (barreiras, determinantes de escolha e uso, necessidades informacionais e velocidade de recuperação).

Os fluxos de informação abordados nesta pesquisa correspondem exclusivamente ao ecossistema comunicativo do campo jornalístico, pois são o fluxo das Fake News. Desde 2000, este ecossistema tem experimentado uma aceleração das mudanças em tecnologia, papéis, novos objetos e atores, razão pela qual tem sido chamado de o "novo 
ecossistema comunicativo". A investigação possui este campo de análise que tem um referencial teórico próprio. Os estudos da Cultura Digital seriam o arcabouço teórico mais abrangente. Com respeito ao âmbito da Comunicação resulta imprescindível a leitura de Castells (2005), para compreender a sociedade em rede e Wolton (2003), que denuncia a utopia da liberdade, solidariedade e fraternidade da rede. Também Andrejevic (2007), que estuda a suposta interatividade e a dupla mão da chamada comunicação digital suscita; e Gillespie (2018), quem procura desmistificar a promessa da objetividade algorítmica; Galindo Cáceres (2008, 2009), para entender a Comunicologia; e Pardo Kuklinski $(2010,2016)$ para abranger o pós-digitalismo. A investigação também deve ter uma abordagem filosófico sociológico desde Foucault (1999), para vincular o poder disciplinar com "a liberdade como condição de relações de poder"; e à sensação de liberdade (que produzem as redes sociais) que Deleuze (1992) chama de "modulação".

O novo ecossistema comunicativo colocou em crise a profissão do jornalista. Portanto, é importante entender que o surgimento das Fake News afetou o trabalho jornalístico e por isso é importante estudar seu fluxo de informações. A este respeito, Messagi Júnior (2019a) identifica seis aspectos da crise do Jornalismo: 1) novo modelo de financiamento; 2) mudanças nas práticas profissionais; 3) mudanças no consumo informativo; 4) a emergência de um novo ecossistema; 5) redesenho da relação com a tecnologia; e 6) demanda de novas competências (profissionais que exigem uma inovadora formação). Com respeito ao ponto 4, a emergência de um novo ecossistema vem crescendo pela Internet, como suporte, e pela digitalização, como linguagem. Isto colocou diversos atores para lutar na mesma arena como os serviços de streaming de música, de filmes e séries, de jogos, de redes sociais etc. As fontes passam a produzir seu próprio conteúdo. Atores políticos disputam a "narrativa" com a mídia tradicional e os novos meios jornalísticos. Aparecem novos atores pseudojornalísticos, parajornalísticos e ativistas digitais. A credibilidade do Jornalismo foi colocada à prova e as Fake News disputam a primazia de dirigir o debate público. O Jornalismo oscila entre se misturar e/ou se hibridizar. Os desafios do novo ecossistema são morais e técnicos. Mas o pesquisador também alerta que se as universidades (na área do jornalismo) não estudam e ensinam essas novas atividades, elas não apenas correm o risco de não conceder competências válidas para a prática profissional; têm um risco maior, o de não ser o 
jornalista um ator social na vida democrática e dar origem a democracias radicalizadas (MESSAGI, 2019b). Portanto, é importante vincular a teoria da informação com a teoria da comunicação.

\section{ESTADO DA ARTE}

Existe uma bibliografia básica com autores como Choo (2003) que estuda como as organizações usam as informações para criar significado, construir conhecimento e tomar decisões; e Davenport e Prusak (1998) para entender ecologia da informação. Por outro lado, são muitos os pesquisadores que dão conta sobre fluxo da informação. No Brasil se destacam as pesquisas desenvolvidas por dois programas de pós-graduação em Ciências da Informação: a Faculdade de Filosofia e Ciências da Universidade Estadual Paulista, com trabalhos de Valentim (2010, 2013a, 2013b) e de Garcia (2012) sobre ambientes e fluxos de informação; e o Centro de Ciências da Educação da Universidade Federal de Santa Catarina, com trabalhos de Inomata (2012) sobre o fluxo da informação tecnológica; Araújo (2014); Inomata e Varvakis (2015); Passos (2012), com o fluxo de informação em projetos de inovação; Inomata, Araújo e Varvakis (2015), com fluxos de informação na perspectiva organizacional em Araújo (2014), e Araújo, Silva e Varvakis (2017). Outros autores que aportam conhecimento sobre o tema são Moraes e Fadel $(2008,2009)$; García e Fadel (2010), com as interferências nos fluxos informacionais; e Guerra (2018), com sua dissertação sobre avaliação do fluxo de produção de materiais informacionais acessíveis na UFRN.

$\mathrm{Na}$ área de Fake News, muitos trabalhos estão sendo desenvolvidos em diferentes ciências e em diferentes lugares do mundo. Cobrir todo o estado da arte neste projeto faria com que a escrita excedesse os limites estabelecidos. No entanto, pode-se sintetizar que existe uma área de estudos sobre Fake News e política desenvolvida principalmente nos Estados Unidos e na Europa; outra área sobre ação jornalística e fact-cheking; em discurso e análise semiótica; aspectos éticos, filosóficos e legais; impactos sociais; da computação: big data, fluxo, comportamento do usuário, disseminação; entre outros. No entanto, do binômio informação-comunicação, com ênfase no fluxo, parece uma perspectiva pouco aprofundada, o que confere maior originalidade ao presente projeto 
de pesquisa. Mas no mesmo trânsito de pesquisa, se encontra o Digital Publics Program, of Digital Media Research Centre, Queensland University of Technology (DMRC QUT, 2020), na Austrália, dirigido por o Professor Axel Bruns. O DMRC QUT está desenvolvendo atualmente trabalhos que podem dialogar com esta investigação. A pesquisa também se enquadra na linha do discurso de ódio nas redes sociais que vem se aprofundando num campo discursivo mais amplo sobre intolerância política, religiosa, aos migrantes, aos povos indígenas, aos negros, à comunidade LGTBQIA+, às minorias em geral ou à simples agressão contra uma pessoa de forma pública virtual. Essa linha também está sendo desenvolvida no Programa de Pós-graduação em Gestão da Informação da Universidade Federal do Paraná (PGGI-UFPR), motivo pelo qual conversa com os trabalhos do Dr. Rodrigo Eduardo Botelho Francisco e seus alunos orientados, entre os quais o doutorando Luiz Rogério Lopes Silva se encontra (SILVA; BOTELHO-FRANCISCO, 2018, 2019).

Por ser a vida dos cidadãos atravessada por essas novas tecnologias, também se enquadra na participação digital, transparência e políticas públicas. Por outro lado, estabelece uma relação entre o ecossistema comunicativo das cidades inteligentes, motivo pelo qual se considera pertinente que a pesquisa dialogue com os temas desenvolvidos no PPGGI- UFPR por meio das pesquisas da Dra. Taine Ritta Coelho. Devido às implicações na vida pública e privada, a pesquisa pode estar inclinada a dialogar com estudos culturais, antropológicos ou sociológicos, mas tomar essa direção significa afastar-se da estrutura definida do binômio informação-comunicação. Alguns aspectos desses estudos já estão contidos nos tópicos abordados pela Dra. Coelho, onde os aspectos jurídicos também podem ser aprofundados.

\section{PERCURSO METODOLÓGICO}

A pesquisa faz parte do Paradigma Construtivista, Naturalista e Interpretativo (HERNÁNDEZ SAMPIERI; FERNÁNDEZ COLLADO; BAPTISTA LUCIO, 2010), pois o estudo não manipula nem estimula variáveis em relação ao fato investigado, que, neste caso, são sujeitos em um contexto específico. Se concentra na compreensão e interpretação de uma situação da realidade. O Construtivismo permite que o pesquisador construa seus próprios procedimentos para resolver uma situação problemática, o que implica que suas 
ideias podem ser modificadas e ele continua aprendendo. O tipo de pesquisa a ser realizada é de metodologia mista, pois serão utilizados alguns dados quantitativos, mas predominam os dados qualitativos, pois uma análise interpretativa dos dados extraídos de uma realidade (concreta e em movimento) é privilegiada.

A Referência empírica tem como universo de estudo o fluxo informacional de Fake News. O corpus ou amostra é o fluxo informacional de Fake News com base em falsas denúncias à justiça. As unidades de observação são as Fake News baseadas em denúncias falsas cujo objetivo era destruir a vida pública e privada das pessoas. Os sujeitos da observação são uma vítima de Fake News por falsas denúncias em Salta, Argentina, e uma vítima em Curitiba, Brasil. Caso 1: Diretor de rádio e chefe de curso na universidade acusado falsamente de abuso sexual. Caso 2: Médica do hospital acusada falsamente de causar a morte de seus pacientes. Os elementos espaço-temporais são: Fake News, denúncias, ações da Justiça, marco jurídico, ecossistema comunicativo, vidas das vítimas desde o aparecimento das notícias falsas e as denúncias até o presente (período 20162020 para o caso 1 e 2013 -2020 para o caso 2 ).

Os métodos e técnicas que serão utilizados para alcançar os objetivos estabelecidos serão: a análise bibliográfica: para definir as unidades conceituais; a análise de documentos: para definir o ecossistema comunicativo e os fatores de fluxo; Análise de conteúdo: para as Fake News e as denúncias falsas; Preparação de mapas conceituais e visuais: para identificação dos fluxos informacionais; a entrevista: de diferentes atores dos fatos; a biografia ou autobiografia: para a apresentação das vítimas; a história da vida: para rastrear a tomada de decisão das vítimas; uma análise semiótica prática: para as notícias, os discursos em circulação e fatores de fluxo; e a triangulação dos dados: para a preparação dos resultados da pesquisa. A possibilidade de outras técnicas etnográficas permanece aberta se o trabalho de campo exigir.

\section{CONSIDERAÇÕES PARCIAIS}

Até novembro de 2020, a pesquisa aprofundou em um aspecto particular que foi a descrição do Ecossistema Comunicativo desde o Fluxo Informacional. Se realizou uma adaptação dos textos de Valentim (2013a, 2013b, 2020) para procurar características do 
Fluxo Informacional em ecossistemas comunicativos concretos nas investigações de Münch (2019) e Quodling (2019), orientados por Bruns (2011). A partir da adaptação de Valetim, foram descritas características do fluxo como: volume, velocidade, variedade, veracidade, valor, volatilidade, validade, variabilidade, visualização de dados que produz, vulnerabilidade de todo tipo que produz, uniformes, não uniformes e mutabilidade.

Em nível geral, se seguirá no trabalho micro sociológico de acordo com o conhecimento prévio que o pesquisador possui e entrará em diálogo com novos conhecimentos aprendidos no PPGGI-UFPR. Possui um olhar teórico interdisciplinar descritivo baseado na área da informação e da comunicação. Pretende-se conhecer certos ecossistemas comunicativos e entender uma situação problemática. Uma possível limitação operacional é a falta de colaboração dos sujeitos envolvidos. Não é realizado um estudo de mineração de dados, de algoritmos porque o pesquisador não possui formação nesse sentido; tampouco são abordadas para a etapa de mestrado, as áreas de text mining, web mining, social mining, mineração de opiniões, análise de sentimentos, mineração de emoções que poderiam estar presentes em estudos posteriores. A pesquisa espera contribuir para aprofundar essa área no programa de pós-graduação, com trabalhos que retornem à sociedade para melhorar a vida dos cidadãos no sistema democrático.

\section{Financiamento}

A pesquisa conta com uma bolsa de estudos da Coordenação de Aperfeiçoamento de Pessoal de Nível Superior (CAPES) fundação do Ministério da Educação (MEC) do Brasil.

\section{REFERÊNCIAS}

ANDREJEVIC, Mark. iSpy Surveillance and Power in the Interactive Era. Lawrence Kansas: University Press of Kansas, 2007.

ARAÚJO, Wánderson Cássio Oliveira. O fluxo de informação em projetos de inovação: estudo em três organizações. 2014. 174 f. Dissertação (Mestrado em Ciência da Informação) - Programa de Pós-graduação em Ciência da Informação, Centro de Ciências da Educação, Universidade Federal de Santa Catarina, Florianópolis, 2014. Disponível em: https://repositorio.ufsc.br/handle/123456789/129178. Acesso em: 24 nov. 2020. 
ARAÚJO, Wánderson Cássio Oliveira; SILVA, Edna Lúcia da; VARVAKIS, Gregório. Fluxos de informação em projetos de inovação: estudo em três organizações. Perspectivas em Ciência da Informação, v. 22, n. 1, p. 57-79, jan./mar. 2017. Disponível em: http://www.scielo.br/scielo.php?script=sci_arttext\&pid=S1413-99362017000100057. Acesso em: 27 out. 2020.

BRUNS, Axel. Gatekeeping, Gatewatching, Realimentação em Tempo Real: novos desafios para o Jornalismo. Brazilian Journalism Research, v. 7, n. 2, p. 119-140, 2011. Disponível em: https://bjr.sbpjor.org.br/bjr/article/view/342. Acesso em: 12 out. 2020.

CASTELLS, Manuel. A sociedade em rede. 8. ed. São Paulo: Paz e Terra, 2005.

CHOO, Chun Wei. A organização do conhecimento: como as organizações usam a informação para criar significado, construir conhecimento e tomar decisões. São Paulo: SENAC Editora, 2003.

DAVENPORT, Thomas H.; PRUSAK Laurence. Ecologia da Informação: por que só a tecnologia não basta para o sucesso na era da informação. Tradução Bernadette Siqueira Abrão. São Paulo: Futura, 1998.

DELEUZE, Gilles. Post-Scriptum sobre as Sociedades de Controle. In: DELEUZE, Gilles. Conversações. Tradução de Peter Pál Pelbart. São Paulo: Editora 34, 1992.

DMRC QUT. Digital Publics. Digital Media Research Centre, Creative Industries Faculty. Queensland University of Technology, Brisbane, Queensland, Australia, 2020. Disponível em: https://research.qut.edu.au/dmrc/programs/. Acesso em: 12 out. 2020.

FOUCAULT, Michel. Vigiar e punir: nascimento da prisão. Petrópolis: Vozes, 1999.

GALINDO CÁCERES, Jesús (coord.). Comunicación, Ciencia e Historia. Fuentes científicas históricas hacia una Comunicología Posible. Madrid: McGraw-Hill/Interamericana de España, 2008.

GALINDO CÁCERES, Jesús. El Campo de la Comunicación y la Comunicología posible. In: CONGRESO REDCOM “Cultura de masas y nuevos procesos de comunicación”, 11.; 2009, Salta. Conferência Magistral. RedCom. Salta: UCASAL, 22, 23, 24 out. 2009.

GARCIA, Regis. Ambientes e fluxos informacionais: modelo de diagnóstico de interferências (DIFI) sob a ótica dos valores culturais. 2012. 365 f. Tese (Doutorado em Ciência da Informação) - Faculdade de Filosofia e Ciências, Universidade Estadual Paulista, Marília, 2012. Disponível em:

https://repositorio.unesp.br/bitstream/handle/11449/103353/garcia_r_dr_mar.pdf;jsessi onid=FCA36AD810DC9C5D0BACA65AC6777591?sequence=1. Acesso em: 24 nov. 2020.

GARCÍA, Regis; FADEL, Bárbara. Interferencias en los flujos informacionales (IFIs): rescate y preservación de la cultura organizacional. Ibersid: revista de sistemas de información y 
documentación, v. 4, p. 211-218, jul. 2010. Disponível em:

https://www.ibersid.eu/ojs/index.php/ibersid/article/view/3852. Acesso em: 27 out. 2020.

GILLESPIE, Tarleton. A relevância dos algoritmos. Parágrafo, São Paulo, Brasil, v. 6, n. 1, p. 95-121, jan./abr. 2018. Disponível em:

http://revistaseletronicas.fiamfaam.br/index.php/recicofi/article/view/722. Acesso em: 24 nov. 2020.

\section{GUERRA, Erica Simony Fernandes de Melo. Gestão da Informação no Laboratório de} Acessibilidade da Biblioteca Central Zila Mamede: avaliação do fluxo de produção de materiais informacionais acessíveis na UFRN. 2018. 187 f. Dissertação (Mestrado Profissional em Gestão da Informação e do Conhecimento) - Programa de Pós-Graduação em Gestão da Informação e do Conhecimento, Centro de Ciências Sociais Aplicadas, Universidade Federal do Rio Grande do Norte, Natal, RN, 2018. Disponível em: https://repositorio.ufrn.br/jspui/bitstream/123456789/25854/1/EricaSimonyFernandesD eMeloGuerra_DISSERT.pdf. Acesso em: 24 nov. 2020.

HERNÁNDEZ SAMPIERI, Roberto; FERNÁNDEZ COLLADO, Carlos; BAPTISTA LUCIO, María del Pilar. Metodología de la investigación. 5. ed. México D.F.: McGraw Hill Interamericana, 2010.

INOMATA, Danielly Oliveira. O fluxo da informação tecnológica: uma análise no processo de desenvolvimento de produtos biotecnológicos. 2012. 283f. Dissertação (Mestrado em Ciência da Informação) - Programa de Pós-Graduação em Ciência da Informação, Centro de Ciências da Educação, Universidade Federal de Santa Catarina, Florianópolis, 2012. Disponível em: https://repositorio.ufsc.br/xmlui/handle/123456789/99498. Acesso em: 27 out. 2020.

INOMATA, Danielly Oliveira; ARAÚJO, Wánderson Cássio Oliveira; VARVAKIS, Gregório. Fluxos de informação na perspectiva organizacional. Informação \& Informação, Londrina, v. 20, n. 3, p. 203-228, set./dez. 2015. Disponível em:

http://www.uel.br/revistas/uel/index.php/informacao/article/viewFile/18209/17645/. Acesso em: 27 out. 2020.

INOMATA, Danielly Oliveira; VARVAKIS, Gregório Jean. A complexidade do fluxo da informação tecnológica e a interação da rede interna no subsidio ao desenvolvimento de produtos biotecnológicos. Revista Biblios, [S.I.], n. 58, p. 1-16, 2015. Disponível em: http://biblios.pitt.edu/ojs/index.php/biblios/article/view/206. Acesso em: 27 out. 2020.

MESSAGI JÚNIOR, Mário. Seis aspectos da crise. Trabalho preliminar do relatório de pesquisa pós-doutoral A crise do Jornalismo. Curitiba: inédito, 2019a.

MESSAGI JÚNIOR, Mário. Outros junhos virão: protestos organizados em rede e as democracias radicalizadas. Curitiba: Kotter Editorial, 2019b. 
MORAES, Cássia Regina Bassan de; FADEL, Bárbara. A informação no contexto organizacional: tipos, características e usos. Revista Ibersid, v. 3, p. 61-65, 2009. Disponível em:

https://edisciplinas.usp.br/pluginfile.php/263594/mod_resource/content/3/Leitura\%20C omplementar_Informa\%C3\%A7\%C3\%A30\%20no\%20Contexto\%20Organizacional.pdf. Acesso em: 27 out. 2020.

MORAES, Cássia Regina Bassan de; FADEL, Bárbara. Perspectivas metodológicas para o estudo da gestão da informação em ambientes informacionais das organizações. Ibersid: revista de sistemas de información y documentación, v. 2, p. 33-41, 2008. Disponível em: https://ibersid.eu/ojs/index.php/ibersid/article/view/2199. Acesso em: 27 out. 2020.

MÜNCH, Felix Victor. Measuring the networked public: Exploring network science methods for large-scale online media studies. 2019. $359 \mathrm{f}$. Thesis (PhD) - Digital Media Research, Centre Creative Industries Faculty, Queensland University of Technology, Brisbane, Queensland, Australia, 2019. Disponível em: https://eprints.qut.edu.au/125543/1/Felix\%20M\%C3\%BCnch\%20Thesis.pdf. Acesso em: 12 out. 2020.

PARDO KUKLINSKI, Hugo. Geekonomía: un radar para producir en el postdigitalismo. Barcelona: Universidad de Barcelona, 2010.

PARDO KUKLINSKI, Hugo. Digithon, Hackathon universitario de Comunicación Digital. In: JORNADAS DE LA RED COBINCO, 5., 2016, Salta. Palestra. Red Cobinco. Salta: UCASAL, 19, 20, 21 maio 2016.

SAUTU, Ruth; BONIOLO, Paula; DALLE, Pablo; ELBERT, Rodolfo. Manual de Metodología: construcción del marco teórico, formulación de los objetivos y elección de la metodología. Buenos Aires: CLACSO, 2005.

SILVA, Luiz Rogério Lopes; BOTELHO-FRANCISCO, Rodrigo Eduardo. A gestão do discurso de ódio nas plataformas de redes sociais digitais: um comparativo entre Facebook, Twitter e Youtube. RICl: Revista Ibero-Americana de Ciência da Informação, Brasília, v. 12, n. 2, p. 470-492, maio/ago. 2019. Disponível em: http://periodicos.unb.br/index.php/RICl/article/view/22025. Acesso em: 26 out. 2020.

SILVA, Luiz Rogério Lopes; BOTELHO-FRANCISCO, Rodrigo Eduardo. Da representação ao monitoramento: a criação de uma ontologia do discurso de ódio online brasileiro. Atoz: novas práticas em informação e conhecimento, v. 7, n. 2, p. 28-33, jul./dez. 2018. Disponível em: https://revistas.ufpr.br/atoz/article/view/67243. Acesso em: 26 out. 2019.

VALENTIM, Marta Lígia Pomim. Ambientes e fluxos de informação em contextos empresariais: o caso do setor cárnico de Salamanca/Espanha. BJIS, Brazilian Journal of Information Science, Marília (SP), v. 7, n. Especial, p. 299-323, 1‥ Sem. 2013a. Disponível em: http://www2.marilia.Unesp.br/revistas/index.php/bjis/index. Acesso em: 27 out. 2019. 
VALENTIM, Marta Lígia Pomim. Ambientes e fluxos de informação: transversalidades. In: SEMINÁRIO TENDÊNCIAS DA GESTÃO DA INFORMAÇÃO EM INSTITUIÇÕES DE CIÊNCIA E TECNOLOGIA, Brasília, 2013b. Disponível em:

http://www.brapci.inf.br/index.php/res/v/3842. Acesso em: 27 out. 2019.

VALENTIM, Marta Lígia Pomim (org.). Gestão, mediação e uso da informação [online]. São Paulo: Editora UNESP, 2010. Disponível em: http://books.scielo.org/id/j4gkh. Acesso em: 27 out. 2019.

VALENTIM, Marta Lígia Pomim. Gestão do Conhecimento e Inteligência social no contexto do Big Data. In: SEMINÁRIO NACIONAL DE GESTÃO DA INFORMAÇÃO E DO CONHECIMENTO, 2., 2020, Natal, RN. Palestra. Rede GIC. Natal: UFRN, 4 set. 2020.

QUODLING, Andrew. Social media governance: Platforms in conflict -the strategies of operators and the tactics of users. 2019. $354 \mathrm{f}$. Thesis (PhD) - Digital Media Research Centre Creative Industries Faculty, Queensland University of Technology, Brisbane, Queensland, Australia, 2019. Disponível em:

https://eprints.qut.edu.au/127473/2/_qut.edu.au_Documents_StaffHome_StaffGroupH \%24_halla_Desktop_Andrew_Quodling_Thesis.pdf. Acesso em: 24 nov. 2020

WOLTON, Dominique. Internet, e depois? Uma teoria crítica das novas mídias. Porto Alegre: Sulina, 2003.

\section{Declaração de Contribuição dos Autores}

Álvaro Maximiliano Pino Coviello - Conceptualização - Curadoria de Dados - Análise Formal - Investigação - Metodologia - Administração do Projeto - Validação Visualização - Escrita (rascunho original) - Escrita (análise e edição).

Rodrigo Eduardo Botelho Francisco - Conceptualização - Metodologia - Administração do Projeto - Supervisão - Validação - Visualização - Escrita (rascunho original) - Escrita (análise e edição). 